\title{
INTERFACE GROWTH DRIVEN BY SURFACE KINETICS AND CONVECTION*
}

\author{
PAK-WING FOK $^{\dagger}$ AND TOM CHOU
}

\begin{abstract}
A moving, solidifying interface that grows by the instantaneous adsorption of a diffusing solute can be described by equations analogous to those of the classical one-sided Stefan problem for solidification. However, the behavior of precipitate growth by material deposition can depend on both surface kinetics and bulk drift of the depositing species. We generalize the Stefan problem and its interface boundary condition to explicitly account for both surface kinetics and particle convection. A surface layer, within which the surface adsorption and desorption kinetics occurs, is introduced. We find that surface kinetics regularizes the divergent interface velocity at short times, while a finite surface layer thickness further regularizes an otherwise divergent initial acceleration. At long times, we find the behavior of the interface position to be governed by the particle drift. The different asymptotic regimes and the cross-over among them are found from numerical solutions of the partial differential equations, as well as from analysis of a nonlinear integro-differential equation.
\end{abstract}

Key words. Stefan problem, kinetics, moving boundary, asymptotics

AMS subject classifications. 74H10, 80A30, 74N20

DOI. $10.1137 / 070710354$

1. Introduction. The process of nucleation and growth due to precipitation of diffusing molecules arises in many physical and biochemical processes. As the precipitate grows, the interface at which adsorption occurs moves. Such moving boundary problems can arise in solidification during phase transitions, step growth in surface physics [4], and even in membrane contact regions between particles and cells [11,6].

The classical moving interface problem, the Stefan problem, describes the growth of a flat interface between solid ice and water, with an accompanying release of heat. Mathematically, the one-sided problem consists of a diffusion equation which is to be solved within a domain $X(t)<x<\infty$, where $X(t)$ is the time-dependent location of the moving boundary. Since $X(t)$ is an unknown, three boundary conditions are required to close the problem. Two conditions are immediately obtained from the far field behavior of the temperature field $T(x, t)$ and a kinematic condition on the interface that governs its velocity (e.g., $\dot{X} \propto \frac{\partial T}{\partial x}$ ). However, deriving the form of the third boundary condition requires additional physical considerations. In the solidification of ice, $T(X, t)=0$, the freezing point of water. This Dirichlet boundary condition is valid as long as the latent heat of fusion is large and the heat capacity of the solid precipitate is small, rendering thermal diffusion the rate-limiting process. Virus wrapping by a cell membrane can also be modeled as a Stefan problem [11]. Here, the contact area between the host cell membrane and a virus particle can be viewed as a growing domain. The concentration of receptors on the cell membrane is governed by diffusion; these receptors bind to ligands on the surface of the virus,

*Received by the editors December 7, 2007; accepted for publication (in revised form) January 12, 2009; published electronically April 15, 2009. This work was supported by National Science Foundation grant DMS-0349195 and National Institutes of Health grant K25AI41935.

http://www.siam.org/journals/siap/70-1/71035.html

${ }^{\dagger}$ Department of Applied and Computational Mathematics, Caltech, Pasadena, CA 91125 (pakwing@caltech.edu), and Department of Biomathematics, UCLA, Los Angeles, CA 90095-1766.

${ }^{\ddagger}$ Department of Mathematics, UCLA, Los Angeles, CA 90095-1555, and Department of Biomathematics, UCLA, Los Angeles, CA 90095-1766 (tomchou@ucla.edu). 
increasing the contact area. Instead of imposing a third boundary condition at the interface, the authors in [11] enforce a global energy dissipation rate that involves cell membrane stiffness, receptor-ligand binding energies, and entropic contributions from receptor molecules.

A simple analytic solution to the classical one-phase Stefan problem is possible with a constant initial condition. In this case, one can show that the interface position is proportional to $\sqrt{t}$. However, this solution predicts an unphysical infinite initial velocity. For the thermal Stefan problem, it has been shown that kinetic undercooling conditions at the interface regularize the solution at $t=0$ [10]. The conditions produce a finite velocity without modifying the heat flux from the interface. However, a similar regularizing condition for material deposition and growth (which is the more relevant physical situation for the one-sided Stefan problem) seems to be lacking.

In surface step growth, a moving boundary problem is frequently used to model a concentration of adsorbed atoms which attach and detach to the edge of one or more growing atomic monolayers [4]. However, in these applications, the concentration of mobile adatoms on the terraces is typically assumed to be equilibrated. This quasisteady approximation neglects transients and is appropriate only at long times, but it simplifies calculations tremendously. Models that assume quasi steadiness have been used to study a wide range of different phenomena including the effects of an applied electric field [22], strain [13], and material deposition [19] on the growth and relaxation of thin films. Models that do not assume quasi steadiness are much less common and seem to focus mainly on growth by deposition [1, 15, 20].

In this paper, we study the one-sided material Stefan problem in the presence of a convective drift and propose a set of boundary conditions that regularize the interface velocity and acceleration at $t=0$. These conditions are analogous to the ones in the thermal Stefan problem [10] but are different both physically and mathematically. This should not be surprising since we have seen (see, e.g., [11]) that closure conditions vary depending on the physical context of the problem being studied. Our proposed boundary condition introduces a thin layer adjacent to the interface within which local attachment and detachment kinetics determine the interface dynamics. Without assuming the quasi-steady approximation, we study the general problem of a precipitate growing under the joint effects of attachment-detachment kinetics, bulk diffusion, and convection.

Our focus is on the one-dimensional problem so that the interface position is described by a single scalar quantity, $X(t)$. The growth of the interface is treated by considering a thin layer with thickness $\varepsilon$ adjacent to $X(t)$ with local concentration $C_{s}(t)$ within and by performing a mass balance. This layer was first introduced in the seminal work of Ward and Tordai [23] as a kinetic "subsurface" layer for the treatment of surfactant $[17,9,18]$ and protein $[3,25]$ adsorption on a static air-water interface. In these applications, the kinetic subsurface layer arises naturally in the expressions connecting surface concentration (number/area) to bulk concentration (number/volume). A physical motivation for this layer based on particle mean free paths or the range of molecular interaction between bulk particles and the surface has been described in [7]. All particles within this layer can adsorb onto the growing interface with rate $k_{\mathrm{on}}$. Similarly, the precipitate can release particles into the layer with rate $k_{\text {off. }}$ This transport due to the "Langmuir kinetics" within the kinetic layer is balanced by convective and diffusive fluxes from the bulk.

The organization of this paper is as follows. In section 2, we include Langmuir kinetics by introducing a modified boundary condition obtained by marrying the subsurface analysis of Ward and Tordai [23] with mass balance at a moving interface. 
After writing the governing equations, we derive an integro-differential equation that describes the motion of the interface. In section 3, we study this equation in the absence of convection and study how the interface motion is modified in the presence of kinetics and a finite kinetic subsurface layer thickness - which we shall call the "layer capacity." Section 4 focuses on the convective case. We show that the inclusion of convection leads to a rich variety of possible behaviors not seen in the classical Stefan problem. We end with a conclusion and discussion in section 5 .

2. Problem setup. Consider a growing interface fed by the deposition of diffusing particles above it. We model this process using three separate regions: a solid region, a microscopically thin kinetic layer, and a bulk region of diffusing and convecting particles. The surface is at position $X(t)$ at time $t$ and grows as particles from the microscopic layer bind to it. This microscopic layer has thickness $\varepsilon$ and lies on top of the surface. We describe the dynamics in a frame moving with velocity $\dot{X}(t)$ such that the solid/kinetic-layer boundary is always at $x=-\varepsilon$ (see Figure 2.1). The kinetic-layer/gas boundary is at $x=0$. Note that in contrast to previous treatments of the Stefan problem, our interface between the solid and gas phases is not a twodimensional plane but a distributed three-dimensional kinetic layer. For the rest of this paper, we shall refer to $x=0$ in Figure 2.1 as "the interface."

The convection diffusion equation describing the bulk concentration for $0<x<$ $\infty$ is

$$
\frac{\partial C}{\partial t}+(U-\dot{X}(t)) \frac{\partial C}{\partial x}=D \frac{\partial^{2} C}{\partial x^{2}},
$$

where $C$ is the bulk particle concentration, $D$ is the bulk diffusivity, and $U$ is an intrinsic drift of the particles participating in precipitation. A physical realization of $U$ could be the electrokinetically driven velocity of charged particles as they precipitate onto an electrode $[2,21]$.

The conservation law for the density of particles in the kinetic layer is

$$
\frac{d}{d t} \int_{-\varepsilon}^{0} C(x, t) d x=k_{\mathrm{off}}-k_{\mathrm{on}} C(-\varepsilon, t)+\left[D \partial_{x} C(x, t)-U C(x, t)\right]_{x=0} .
$$

Within sufficiently thin kinetic layers, we can neglect spatial variations in $C(x, t)$ and write $C(x, t)=C_{s}(t)$ whenever $-\varepsilon<x<0$ and where $\lim _{x \rightarrow 0^{+}} C(x, t)=C_{s}(t)$. Thus, we obtain a boundary condition for (2.1) at $x=0$ :

$$
\varepsilon \frac{\partial C_{s}(t)}{\partial t}=k_{\mathrm{off}}-k_{\mathrm{on}} C_{s}(t)+\left.D \frac{\partial C}{\partial x}\right|_{x=0}-U C_{s}(t)
$$

In (2.3), we are assuming that particles entering the kinetic layer through diffusive and convective fluxes are immediately free to participate in kinetic attachment and detachment, characterized by rates $k_{\text {on }}$ and $k_{\text {off }}$. The intrinsic adsorption speed $k_{\text {on }}$ has units of length per time and $k_{\text {off }}$ is the intrinsic desorption rate per unit area. Also note that both $C_{s}(t)$ in (2.3) and $C(x, t)$ used in (2.1) and (2.2) describe particle densities in a frame moving with the interface.

The rate of binding of particles onto the surface is proportional to the concentration in the thin layer, $C_{s}(t)$, while the desorption of particles from the precipitate into the layer occurs spontaneously with rate $k_{\text {off }}$ : see the inset in Figure 2.1. Therefore, the kinematic condition for the interface is

$$
\dot{X}(t)=v_{0}\left(k_{\mathrm{on}} C_{s}(t)-k_{\mathrm{off}}\right),
$$

Copyright (c) by SIAM. Unauthorized reproduction of this article is prohibited. 


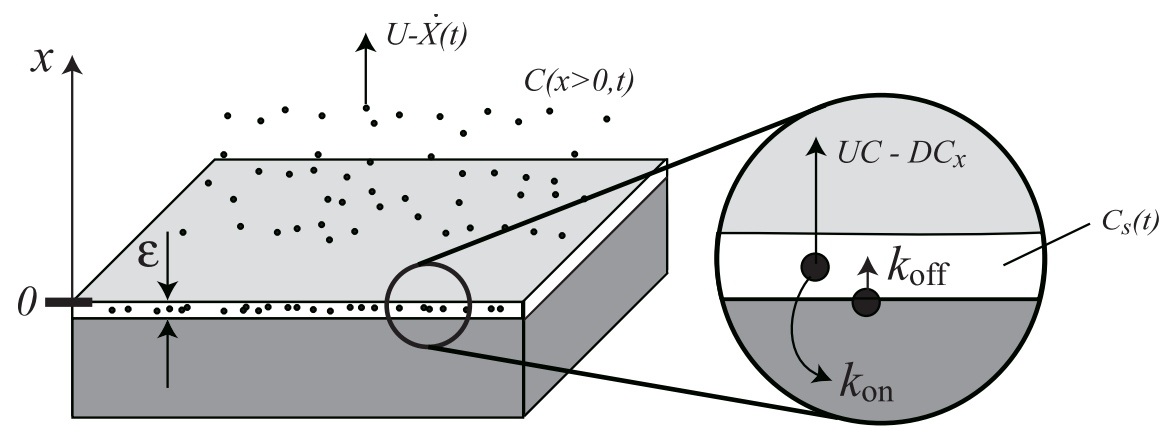

FIG. 2.1. The growth of an interface due to particle adsorption, viewed in the reference frame of the interface. In the bulk phase particles at concentration $C(x, t)$ diffuse with diffusivity $D$ and move with drift velocity $U-\dot{X}$. A layer of thickness $\varepsilon$ below $x=0$ is introduced. Within this layer, surface kinetics characterized by particle attachment and detachment parameters $k_{\mathrm{on}}$ and $k_{\mathrm{off}}$ occurs.

where $v_{0}$ is the molecular volume of the outermost layer of adsorbed particles. Finally, we have the far field condition for $C(x, t)$

$$
\lim _{x \rightarrow \infty} C(x, t)=C_{0} .
$$

Note that unlike the usual Stefan problem, (2.3) and (2.4) do not fix the concentration at the interface: rather it is determined by kinetics and is in general time-dependent. The boundary condition (2.3) includes the effects of a convective drift and a diffusive flux into the kinetic layer [12]. Similar conditions that include kinetics in the Stefan problem were used in [14], [8], and [10].

We nondimensionalize length by $\left(D / k_{\text {off }}\right)^{1 / 4}$ and time by $\left(D k_{\text {off }}\right)^{-1 / 2}$, defining new independent variables

$$
\bar{x}=\frac{x}{\left(D / k_{\mathrm{off}}\right)^{1 / 4}}, \quad \bar{t}=\frac{t}{\left(D k_{\mathrm{off}}\right)^{-1 / 2}},
$$

new dependent variables

$$
\bar{\varphi}=v_{0}\left(C-\frac{k_{\mathrm{off}}}{k_{\mathrm{on}}}\right), \quad \bar{X}=\frac{X}{\left(D / k_{\mathrm{off}}\right)^{1 / 4}},
$$

and new dimensionless parameters

$$
\begin{aligned}
& \bar{\varepsilon}=\frac{\varepsilon}{\left(D / k_{\mathrm{off}}\right)^{1 / 4}}, \quad \eta=\frac{D^{3 / 4} k_{\mathrm{off}}^{1 / 4}}{k_{\mathrm{on}}}, \\
& \bar{v}_{0}=\frac{v_{0}}{\left(k_{\mathrm{on}} / k_{\mathrm{off}}\right)}, \quad \bar{U}=\frac{U}{D^{3 / 4} k_{\mathrm{off}}^{1 / 4}},
\end{aligned}
$$

so that the bulk equation (2.1) satisfied on $0<\bar{x}<\infty$ becomes

$$
\frac{\partial \bar{\varphi}}{\partial \bar{t}}+\left(\bar{U}-\frac{d \bar{X}}{d \bar{t}}\right) \frac{\partial \bar{\varphi}}{\partial \bar{x}}=\frac{\partial^{2} \bar{\varphi}}{\partial \bar{x}^{2}}
$$


the boundary conditions (2.3) and (2.4) at $\bar{x}=0$ become

$$
\begin{aligned}
\bar{\varepsilon} \eta \frac{d \bar{\varphi}_{s}}{d \bar{t}} & =-\bar{\varphi}_{s}+\left.\eta \frac{\partial \bar{\varphi}}{\partial \bar{x}}\right|_{\bar{x}=0}-\eta \bar{U} \bar{\varphi}_{s}-\eta \bar{v}_{0} \bar{U}, \\
\frac{d \bar{X}}{d \bar{t}} & =\frac{\bar{\varphi}_{s}}{\eta}
\end{aligned}
$$

and the far field condition is

$$
\lim _{\bar{x} \rightarrow \infty} \bar{\varphi}(\bar{x}, \bar{t})=\bar{\varphi}_{0}
$$

In $(2.10)$ and $(2.11), \bar{\varphi}_{s}(\bar{t}) \equiv \bar{\varphi}(0, \bar{t})$. In this paper, we solve $(2.9)-(2.12)$ with the initial condition $\bar{\varphi}(\bar{x}, 0)=\bar{\varphi}_{0}$, a constant. If $C_{0}$ is the initially uniform particle concentration, $v_{0} C_{0}$ represents the initial volume fraction of the solute and must therefore lie between 0 and 1 so that

$$
-\bar{v}_{0}<\bar{\varphi}_{0}<1-\bar{v}_{0}
$$

where $\bar{v}_{0}>0$. As will be shown, this physical constraint on the initial condition will rule out solutions to our problem that would otherwise be mathematically correct. The constraint (2.13) does not arise in the thermal Stefan problem.

When $\bar{U}=0$ and $\bar{\varepsilon}=0,(2.9)-(2.12)$ become mathematically identical to the thermal undercooling problem studied in [8] and [14], with $\eta$ playing the role of the undercooling parameter. In this case, analytic long time similarity solutions are possible $[8,14]$. However, these solutions are valid only when $\bar{\varphi}_{0}<1$ in the far field. The finite time singularities and traveling waves which arise for $\bar{\varphi}_{0}>1$ are not present in our material problem because of the constraint (2.13).

Our focus in this paper will be on how the values of $\eta, \bar{\varepsilon}$, and $\bar{U}$ affect the motion of the interface. We now drop all overbars to simplify the notation with the understanding that all variables and parameters are dimensionless for the rest of this paper. Qualitatively, $\varepsilon$ describes the capacity for addition of particles into the kinetic layer. For example, when $\varepsilon=0$, the increase in the number of atoms in the layer due to the convective and diffusive fluxes is always equal to the number of atoms leaving the layer due to the interfacial kinetics. Henceforth, we shall refer to $\varepsilon$ as the "layer capacity." The parameter $\eta$ describes the speed of diffusion relative to the speed of attachment, and $U$ is the nondimensional convection velocity. Upon substituting (2.10) into (2.11), we have

$$
\dot{X}=\left.\left[\frac{\partial \varphi}{\partial x}-\varepsilon \frac{d \varphi_{s}}{d t}-\varphi U-v_{0} U\right]\right|_{x=0}
$$

Physically, we expect the behavior of the system to approach that of the classical Stefan problem when $k_{\text {on }}$ is large and $U$ and $\varepsilon$ are small in (2.8), obtained by letting $\eta, \bar{\varepsilon}, \bar{U} \rightarrow 0$. In fact, the usual Stefan conditions for the one-phase problem $\varphi_{s}=$ $0, \dot{X}=\left.\frac{\partial \varphi}{\partial x}\right|_{x=0}$ are obtained from (2.14) and (2.10) when these limits are taken. From inspection of (2.10), we suspect that the $\eta \rightarrow 0$ limit may be a singular one since $\eta$ multiplies the highest time derivative in the boundary condition. 
The solution for $\varphi$ can be found by taking Laplace transforms in $x$ of (2.9) and $(2.10)$ :

$$
\begin{aligned}
\varphi(x, t) & =\frac{\varphi_{0}}{2}\left[1+\operatorname{erf}\left(\frac{x-U t+X(t)}{2 \sqrt{t}}\right)\right] \\
& -\frac{1}{2 \sqrt{\pi}} \int_{0}^{t} \frac{\mathrm{d} \tau}{\sqrt{\tau}} e^{-(x+X(t)-X(t-\tau)-U \tau)^{2} / 4 \tau}\left[v_{0} U+\frac{1}{\eta} \varphi_{s}(t-\tau)\right. \\
& \left.+\left(\dot{X}(t-\tau)-\frac{x+X(t)-X(t-\tau)-U \tau}{2 \tau}\right) \varphi_{s}(t-\tau)+\varepsilon \dot{\varphi}_{s}(t-\tau)\right] .
\end{aligned}
$$

By carefully taking the limit $x \rightarrow 0$ (see Appendix, section 6.1) and substituting $\dot{X}=\varphi_{s}(t) / \eta$, we obtain an integro-differential equation for $X(t)$ :

$$
\begin{aligned}
\eta \dot{X}(t) & =\varphi_{0}\left[1+\operatorname{erf}\left(\frac{X(t)-U t}{2 \sqrt{t}}\right)\right] \\
& -\frac{1}{\sqrt{\pi}} \int_{0}^{t} \frac{\mathrm{d} \tau}{\sqrt{\tau}} e^{-(U \tau-X(t)+X(t-\tau))^{2} / 4 \tau}\left[v_{0} U+\dot{X}(t-\tau)\right. \\
& \left.+\eta\left(\dot{X}(t-\tau)-\frac{X(t)-X(t-\tau)-U \tau}{2 \tau}\right) \dot{X}(t-\tau)+\varepsilon \eta \ddot{X}(t-\tau)\right] .
\end{aligned}
$$

In principle, $(2.16)$ can be solved to find $X(t)$, which can then be substituted back into (2.15) to recover $\varphi(x, t)$. Although we are not able to solve (2.16) analytically, we can use it to extract the asymptotic behavior of $X(t)$ in certain cases. When $\eta \ll 1$, we expect that there will be a transient (boundary layer in time) near $t=0$. Within this layer, the layer capacity and the kinetics will play important roles. Outside of this boundary layer, the motion of the interface should be well approximated by the exact solution of the classical one-sided Stefan problem when $U=0$.

3. Nonconvective case. First assume $\eta \ll 1$. The outer solution valid for long times is obtained by neglecting all terms proportional to $\eta$ in (2.16). The resulting equation is

$$
\varphi_{0}\left[1+\operatorname{erf}\left(\frac{X(t)}{2 \sqrt{t}}\right)\right]=\frac{1}{\sqrt{\pi}} \int_{0}^{t} \frac{\mathrm{d} \tau}{\sqrt{\tau}} e^{-[X(t-\tau)-X(t)]^{2} / 4 \tau} \dot{X}(t-\tau) .
$$

We seek a solution of the form $X(t)=X_{\text {out }}(t)=2 \alpha \sqrt{t}$ and obtain

$$
\begin{aligned}
\varphi_{0}[1+\operatorname{erf}(\alpha)] & =\frac{\alpha}{\sqrt{\pi}} \int_{0}^{1} \mathrm{~d} y \frac{e^{-\alpha^{2}(\sqrt{1 / y}-\sqrt{1 / y-1})^{2}}}{\sqrt{y} \sqrt{1-y}}, \\
& =\frac{4 \alpha}{\sqrt{\pi}} \int_{0}^{1} \mathrm{~d} \xi \frac{e^{-\alpha^{2} \xi^{2}}}{1+\xi^{2}}, \\
& =\sqrt{\pi} \alpha e^{\alpha^{2}}\left(1-\operatorname{erf}^{2}(\alpha)\right) .
\end{aligned}
$$

Therefore $\alpha$ satisfies the familiar Stefan transcendental equation [5]:

$$
\alpha=\frac{\varphi_{0}}{\sqrt{\pi}} \frac{e^{-\alpha^{2}}}{\operatorname{erfc}(\alpha)} .
$$

Copyright $@$ by SIAM. Unauthorized reproduction of this article is prohibited. 
We conclude that the outer solution to (2.16) in the absence of convection is exactly the one predicted by the classical Stefan problem: for sufficiently long times, the motion of the interface is limited by diffusion and kinetics is relatively unimportant.

Upon defining $\delta \equiv \varepsilon / \eta$, the short time behavior of (2.16) can be extracted by setting $\xi=t / \eta^{2}, \xi^{\prime}=\tau / \eta^{2}$, and $X(t)=\eta Y(\xi)$ :

$$
\begin{aligned}
Y^{\prime}(\xi)= & \varphi_{0}\left[1+\operatorname{erf}\left(\frac{Y(\xi)}{2 \sqrt{\xi}}\right)\right]-\frac{1}{\sqrt{\pi}} \int_{0}^{\xi} \frac{d \xi^{\prime}}{\sqrt{\xi^{\prime}}} e^{-\left[Y(\xi)-Y\left(\xi-\xi^{\prime}\right)\right]^{2} / 4 \xi^{\prime}} \\
& \times\left[Y^{\prime}\left(\xi-\xi^{\prime}\right)+Y^{\prime}\left(\xi-\xi^{\prime}\right)\left(Y^{\prime}\left(\xi-\xi^{\prime}\right)-\frac{Y(\xi)-Y\left(\xi-\xi^{\prime}\right)}{2 \xi^{\prime}}\right)+\delta Y^{\prime \prime}\left(\xi-\xi^{\prime}\right)\right] .
\end{aligned}
$$

We seek series solutions of the form

$$
Y(\xi) \sim \sum_{m=1}^{\infty} c_{m} \xi^{m / 2}
$$

valid for $\xi \ll 1$. In the limit of vanishing layer capacity $\delta \equiv \varepsilon / \eta=0$, we easily find the first three coefficients $c_{1}=0, c_{2}=\varphi_{0}$, and $c_{3}=-4 \varphi_{0} /(3 \sqrt{\pi})$. Therefore, for small times we obtain

$$
\frac{X(t)}{\eta} \sim \varphi_{0}\left(\frac{t}{\eta^{2}}-\frac{4}{3 \sqrt{\pi}}\left(\frac{t}{\eta^{2}}\right)^{3 / 2}+\ldots\right) .
$$

This behavior is confirmed numerically in Figure 3.1(a). Details of the numerical procedure are given in Appendix, section 6.2. From (3.6), we see that $\dot{X}(0)$ is finitethe inclusion of kinetics has regularized the velocity at $t=0$ : recall that the outer solution $X_{\text {out }}(t)=2 \alpha \sqrt{t}$ predicts that $\dot{X}_{\text {out }}(t) \rightarrow \infty$ as $t \rightarrow 0$. However, because of the $t^{3 / 2}$ term in (3.6), the acceleration of the interface still diverges for small $t$.

Now consider the case where $\delta=\varepsilon / \eta>0$. We have $c_{2}=\varphi_{0}$ as before, but in this case $c_{3}=0$ and $c_{4}=-\varphi_{0} /(2 \delta)$. Therefore, the solution for $\varepsilon>0$ takes the form

$$
X(t) \sim \varphi_{0}\left[\frac{t}{\eta}-\frac{1}{2 \varepsilon}\left(\frac{t}{\eta}\right)^{2}+\ldots\right] .
$$

Hence the inclusion of a nonzero capacity in the kinetic layer has regularized the acceleration of the interface. Numerical data of $\dot{X}(t)$ versus $t$ is shown in Figure 3.1(b) along with the analytic prediction (3.7). Note that $\ddot{X}(0)$, given by the slope at $t=0$, is finite but increases in magnitude as $\varepsilon \rightarrow 0$. Clearly, $X(t)$ cannot have a finite acceleration if the interface velocity is infinite. This fact is reflected in the structure of (2.10) and (2.16), where $\varepsilon$ always multiplies $\eta$. One can regularize the acceleration by taking $\varepsilon>0$ only if $\eta>0$.

In summary, the addition of kinetics has regularized the velocity, $\dot{X}$, through (3.6) and the addition of layer capacity has regularized the acceleration, $\ddot{X}$, through (3.7). The interface position is, of course, a physical quantity and should be an analytic function of $t$ : it should have a Taylor expansion about $t=0$ containing only integer powers, corresponding to $c_{m}=0$ when $m$ is odd in (3.5). However, although the first two terms of (3.7) are integer powers ( order terms are also integer powers. In other words, we do not know if the inclusion of a finite kinetic layer capacity results in $c_{m}=0$ when $m$ is odd and $>3$. 

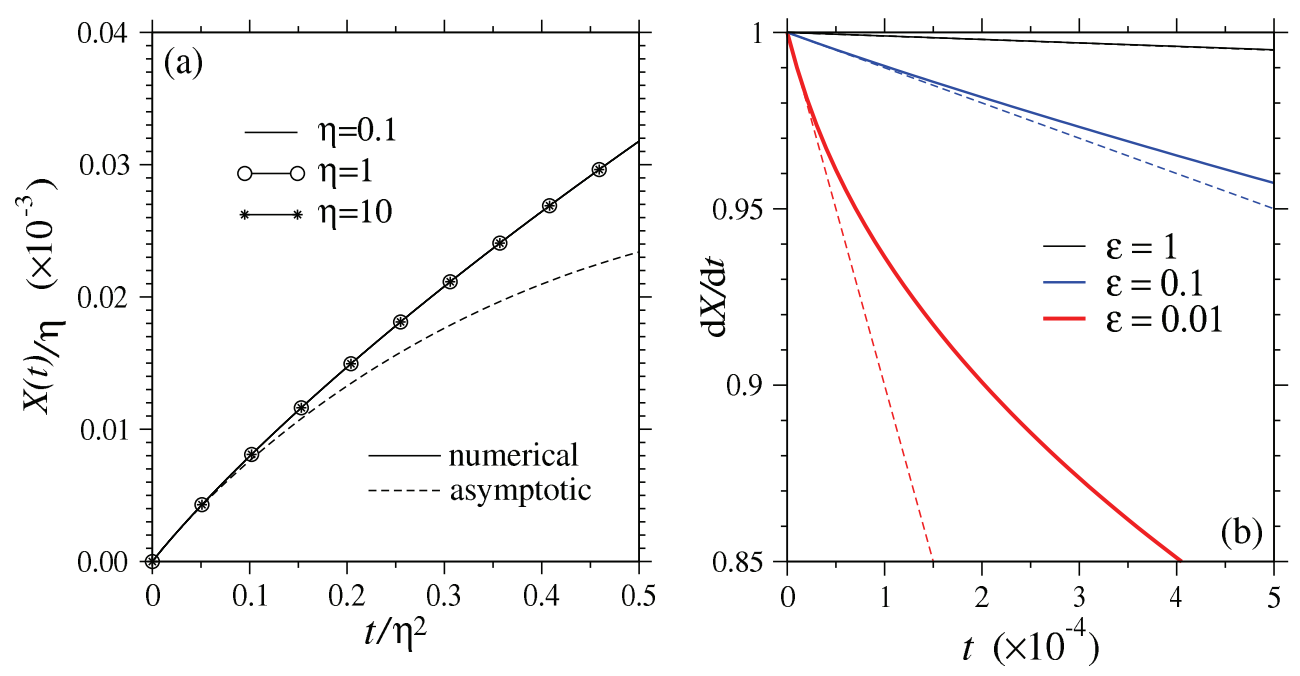

FIG. 3.1. (a) Short time behavior of the interface position. Solid lines indicate the numerical solution to the full problem (with $\varepsilon=U=0, v_{0}=0.5$, and $\varphi_{0}=0.1$ ) and dashed lines show the asymptotic solution given by (3.6). Note that $\eta$ is scaled out of the problem. (b) Data from numerical experiments (solid curves) with $\eta=0.1, U=0, v_{0}=0.5$, and $\varphi_{0}=0.1$ confirms the relation $\dot{X} \sim \varphi_{0}\left(\frac{1}{\eta}-\frac{t}{\varepsilon \eta^{2}}\right)$ in (3.7) (dashed curves). The agreement is better for larger values of $\delta \equiv \varepsilon / \eta$. Larger values of surface layer capacity result in a slower precipitation rate, yielding slower interface growth.

4. The effect of convection. In this section, we focus on the long time behavior of the interface when $U \neq 0$. We assume that both $\varepsilon$ and $\eta$ are small but nonzero.

4.1. Positive convection. The addition of convection in the bulk phase leads to an interesting qualitative change in the behavior of the interface. We expect that the addition of even a small amount of convection will affect $X(t)$ significantly for large times. Assume that bulk particles drift away from the interface so that $U>0$. Furthermore, assume that $\varphi_{0}>0$. For intermediate times, the diffusive flux into the interface will dominate convection so that the interface will grow, i.e., $\dot{X}>0$. However, for longer times, convection will become more important and start depleting the concentration in the kinetic layer. This will eventually result in $\dot{X}<0$, as shown by the thick black curves in Figure 4.1(a). We define the time at which $\dot{X}$ starts to become negative to be $t^{*}$ so that $\dot{X}\left(t^{*}\right)=0$. This defines $X^{*} \equiv X\left(t^{*}\right)$ as the maximum interface position attained. The position of the interface will start to retreat when convection becomes comparable to the effect of diffusion, i.e., $\sqrt{t^{*}} \sim U t^{*} \Rightarrow t^{*} \sim 1 / U^{2}$, $X^{*} \sim \sqrt{t^{*}} \sim 1 / U$. This scaling is confirmed by data from numerical experiments, shown in Figure 4.1(b), and breaks down when $\eta U \geq O(1)$. In this case, strong convection precludes diffusive behavior and the system transitions directly from the kinetic to the convective regime.

From Figure 4.1(a), we notice that for long times, the motion of $X(t)$ is approximately linear. We postulate that for long times and $U>0$, the interface moves with a constant negative velocity, driven by the bulk convection. With this in mind, we take $X(t)=V t$ for some constant $V<0$ and substitute this ansatz into (2.16). As $t \rightarrow \infty$, the solution becomes independent of $\varphi_{0}$ and we obtain $V=-\frac{v_{0} U}{1+\eta U}$ so that

$$
X(t) \sim-\left(\frac{v_{0} U}{1+\eta U}\right) t, \quad t \gg \frac{1}{U^{2}} .
$$



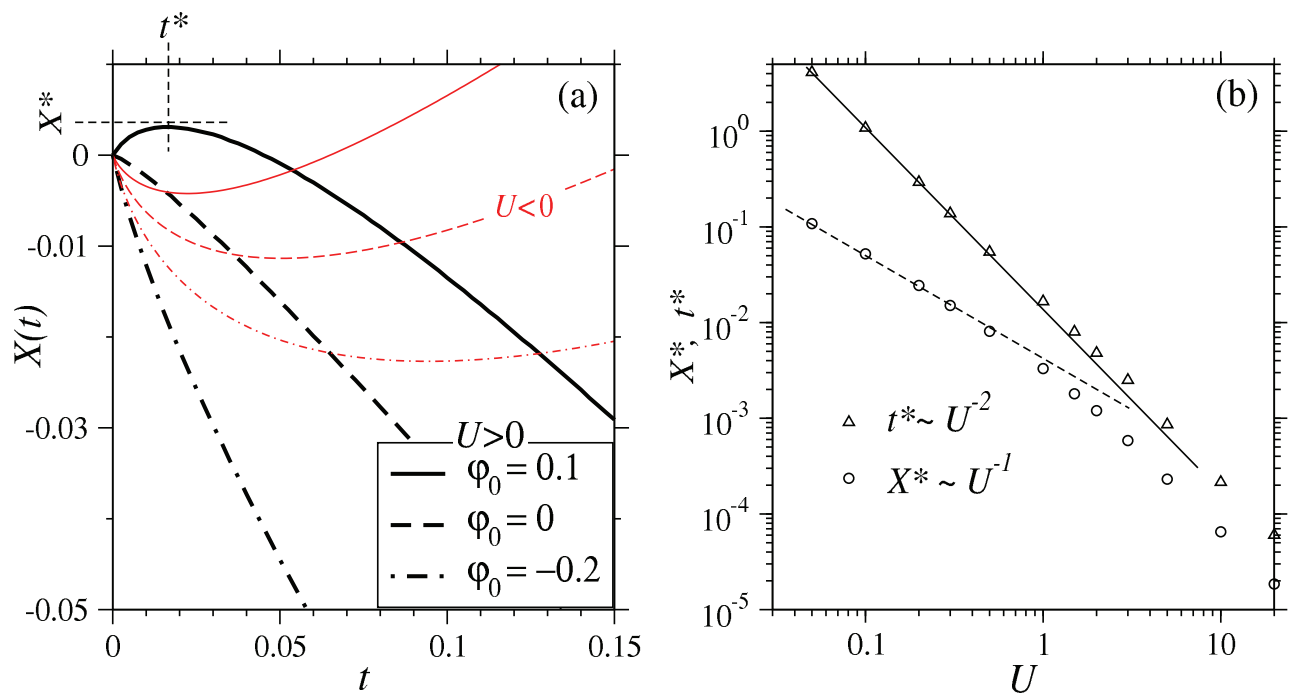

FIG. 4.1. (a) When $U>0$ (thick curves), the long time behavior for $X(t)$ is given by (4.1). For $\varphi_{0}>0$, the behavior of $X(t)$ is nonmonotonic if any amount of positive convection is present. When $\varphi_{0}<0, \dot{X}<0$ for all time. Parameters used were $\eta=0.1, \varepsilon=0, U=1$, and $v_{0}=0.5$. When $U<0$ (thin curves), $X(t)$ can also be nonmonotonic and the long time velocity is given by (4.3). For $U<0$, the parameters used were $\eta=0.1, \varepsilon=0, U=-1$, and $v_{0}=0.5$. The initial densities were $\varphi_{0}=-0.1$ (solid), $\varphi_{0}=-0.15$ (dashed), and $\varphi_{0}=-0.2$ (dot-dashed). (b) Numerical experiments confirm the scaling behaviors $t^{*} \sim 1 / U^{2}$ and $X^{*} \sim 1 / U$, valid when $U \ll 1 / \eta$. Parameters used were $\eta=0.1, \varepsilon=0, v_{0}=0.5$, and $\varphi_{0}=0.1$.

In cases where attachment rates are much larger than detachment rates so that $\eta, v_{0} \ll$ 1 , we can use (4.1) as the long time solution for $\dot{X}$ to see that $\varphi_{s}=-\eta v_{0} U /(1+\eta U)=$ $O\left(\eta v_{0}\right)$. The linear behavior in $X(t)$ arises sooner when $U$ is large. Therefore, when $U \gg 1$, we substitute $X(t)=V t$ into $(2.15)$ to obtain the bulk concentration

$$
\varphi(x, t)=\frac{\varphi_{0}}{2}\left[1+\operatorname{erf}\left(\frac{x-(U-V) t}{2 \sqrt{t}}\right)\right]+O\left(\eta, v_{0}\right) .
$$

Equation (4.2) has a simple physical interpretation. It describes particles diffusing and advecting away from the interface with velocity $U$, but viewed in a frame of reference that moves with velocity $V$ (see Figure 4.2(a)).

4.2. Negative convection. Now let us consider the case where $U<0$. Again, we look for a long time linear solution $X(t \gg 1) \sim V t$ to (2.16), where $V$ must be $>0$. We find that $V$ satisfies $\eta V^{2}+\left(1-\varphi_{0}\right) V+U\left(v_{0}+\varphi_{0}\right)=0$ so that

$$
V=-\frac{1}{2 \eta}\left(1-\varphi_{0}\right)+\frac{1}{2 \eta}\left[\left(1-\varphi_{0}\right)^{2}-4 \eta U\left(\varphi_{0}+v_{0}\right)\right]^{1 / 2},
$$

and we have taken the positive root. Taking the negative root always gives a negative value for $V$ which is unphysical. Note that $4 \eta U\left(\varphi_{0}+v_{0}\right)<0$ because of $(2.13)$. Mathematically, it is possible to have both $U, V<0$ by choosing $\left(\varphi+v_{0}\right)<0$, but these solutions are not physically meaningful in the context of surface growth.

Although the long time velocity $V>0$ in (4.3), at short times kinetic detachment or diffusive evaporation can dominate, giving rise to a transient negative velocity as shown by the thin curves in Figure 4.1(a). For a precipitate of finite thickness, this 

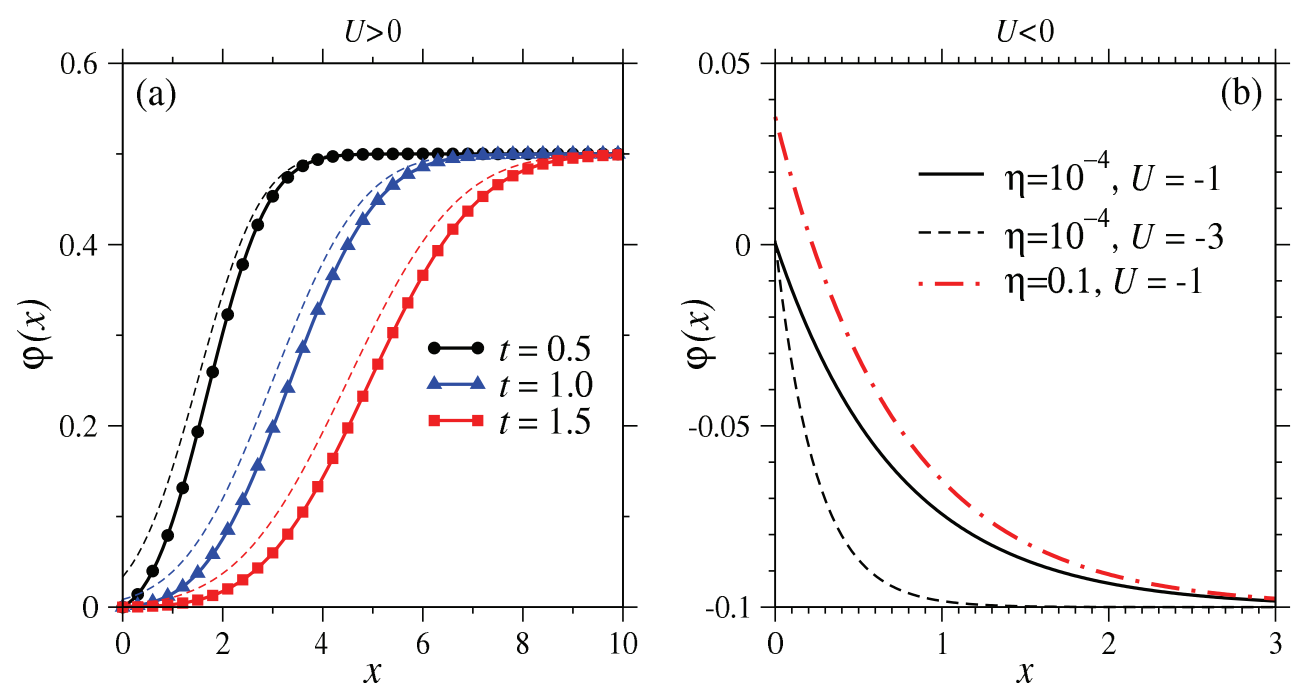

FIG. 4.2. (a) When $U>0$, the long time solution for $\varphi(x, t)$ is a diffusing wave. Solid lines with symbols show data from simulation and corresponding dashed lines indicate the small $\eta$ approximation given by (4.2). Parameters used for the numerical experiments were $\eta=10^{-4}, \varepsilon=0$, $U=3, v_{0}=10^{-4}$, and $\varphi_{0}=0.5$. (b) When $U<0$, steady state solutions are possible, given by (4.4). Here, we used $\varepsilon=0, v_{0}=0.5$, and $\varphi_{0}=-0.1$.

transient dissolution can result in complete annihilation of the precipitate before the onset of $\dot{X}>0$ growth.

Surprisingly, unlike the $U>0$ case, the bulk excess volume fraction $\varphi(x, t)$ corresponding to the interface velocity (4.3) is time-independent and found from integrating (2.9) directly:

$$
\varphi(x)=\varphi_{0}+\left(\varphi_{s}-\varphi_{0}\right) e^{-(V-U) x}
$$

where $\varphi_{s}=\eta V=-\frac{1}{2}\left(1-\varphi_{0}\right)+\frac{1}{2}\left[\left(1-\varphi_{0}\right)^{2}-4 \eta U\left(\varphi_{0}+v_{0}\right)\right]^{1 / 2}$ and $V$ is given by (4.3). The inclusion of a negative convection causes the interface to eventually grow linearly with time, resulting in steady state solutions. The steady concentration profile from (4.4) is shown in Figure 4.2(b) and was verified numerically. Note that we require $V-U>0$ for this solution to be valid. For more negative $U$, the particles are pushed faster against the growing interface, resulting in a thinner boundary layer in $\varphi(x)$. The surface density $\varphi_{s}$ is unaffected by $U$ and depends instead on the surface kinetics through $\eta$. As the effective surface adsorption rate decreases, $\eta$ increases, thereby increasing the surface density $\varphi_{s}$.

From the preceding analyses, it is clear that the $\varphi_{0}, U>0$ and $\varphi_{0}, U<0$ cases are not "symmetric": the transformations $U \rightarrow-U, \varphi_{0} \rightarrow-\varphi_{0}, X \rightarrow-X$ do not leave (2.9)-(2.12) invariant. For any value of $U \neq 0$, we expect eventually a linear behavior $X(t) \sim V t$. The dependence of $V$ on $U$ is shown in Figure 4.3. Interestingly, when $U<0$, the prefactor $V$ depends explicitly on the initial condition, but this is no longer true when $U>0$. When $U<0$, bulk particles with a concentration determined by $\varphi_{0}$ are convected into the interface and one would expect the interface velocity to be $\varphi_{0}$-dependent. On the other hand, when $U>0$, bulk particles are convected away from the surface and therefore the long time interface velocity is independent of $\varphi_{0}$.

In summary, for $\eta U \ll 1$, we have identified three different regimes for $X(t)$, depending on which of the three physical effects (kinetics, diffusion, convection) gov- 


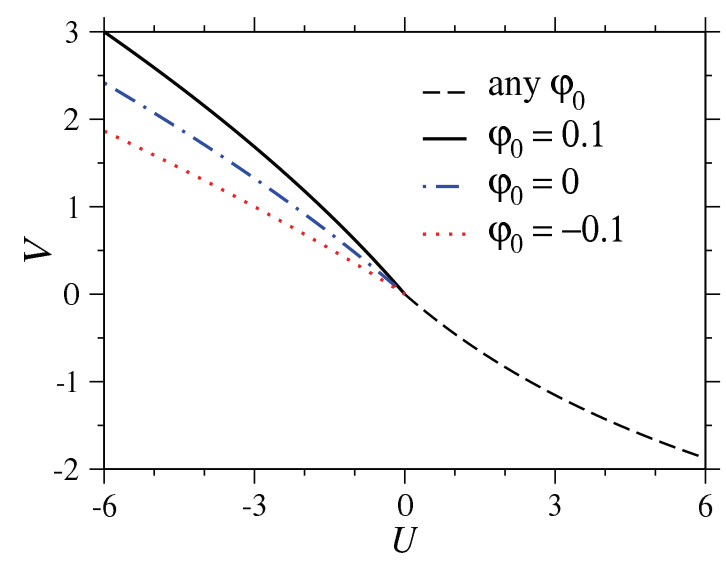

FIG. 4.3. Dependence of the prefactor $V$ on $U$, where $X(t) \sim V t, t \gg 1$. When $U>0, V$ is given by (4.1), which is valid for positive and negative $\varphi_{0}$. When $U<0, V$ is given by (4.3). At $U=0$, no linear behavior is possible and $V$ is undefined. Parameters used were $\eta=0.1, \varepsilon=0$, and $v_{0}=0.5$.
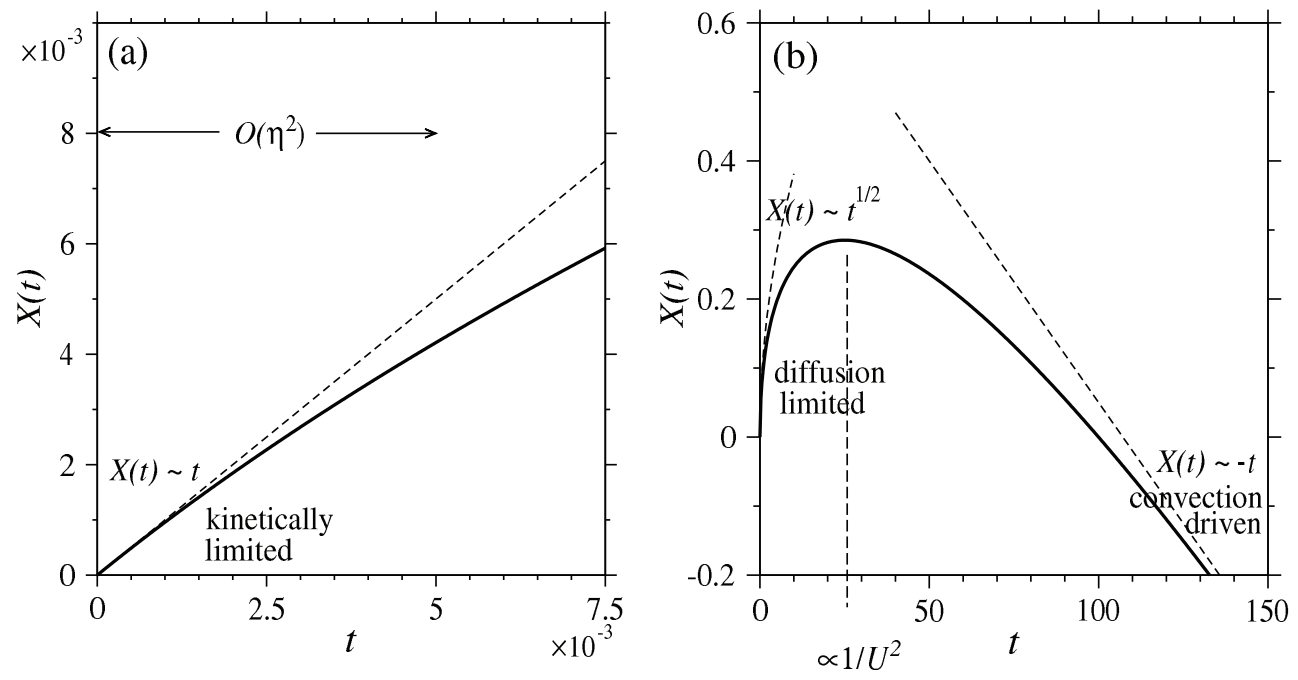

FIG. 4.4. The three types of behavior when $\varepsilon=0$ and $U>0$. (a) At early times, $t \ll \eta^{2}$, attachment-detachment kinetics dominates and $X(t) \sim t$. Parameters used were $\eta=0.1, \varepsilon=0.1$, $U=0.02, v_{0}=0.5$, and $\varphi_{0}=0.1$. (b) At intermediate times, the growth is diffusion-limited and $X(t) \sim \sqrt{t}$ as in the classical Stefan problem. At long times, bulk convection drives the interface growth and $X(t) \sim-t$. Parameters used were $\eta=0.1, \varepsilon=0.1, U=0.02, v_{0}=0.5$, and $\varphi_{0}=0.1$.

erns the dynamics. When $t \ll O\left(\eta^{2}\right)$, we have a kinetics dominated regime where $X(t)=O(t)$. When $O\left(\eta^{2}\right) \ll t \ll O\left(1 / U^{2}\right)$, diffusion dominates the motion of the interface and $X(t) \sim \sqrt{t}$. Finally, convection dominates when $t \gg O\left(1 / U^{2}\right)$, leading to a (negative) linear behavior, $X(t)=O(-t)$. These three regimes are shown in Figure 4.4. We have thus far considered only $\eta U \ll 1$. For $\eta U \geq O(1)$, we expect that the convection regime will arise at a shorter time, shrinking the duration of the diffusive regime. Our analyses in subsection 4.1 will no longer be valid for large $\eta U$, as shown in Figure 4.1(b). 
5. Discussion and conclusions. In this paper we studied the Stefan problem but with the inclusion of three additional physical effects: interface kinetics, layer capacity, and convective drift. We derived a nonlinear integro-differential equation which, in principle, can be solved to find the interface position at any time. In the special case where kinetics, capacity, and drift are negligible, we recovered the solution to the classical problem. The solution to the classical problem has a specific physical meaning in the context of surface growth when convection is absent: it is the "outer" solution to a boundary layer problem described by (2.16). This outer solution $X_{\text {out }}(t)$ is valid for sufficiently long times, $t \gg \eta^{2}$, where $\eta$ describes the speed of diffusion relative to the speed of attachment.

We analyzed the integro-differential equation (2.16) for short times and found that the inclusion of kinetics and kinetic layer capacity in the interface condition rendered the velocity and acceleration of the interface finite. On a related note, the classical Stefan problem does not admit a smooth, uniform concentration as its initial condition. Because $\dot{X}_{\text {out }}(0)$ is infinite, $\left.\frac{\partial \varphi}{\partial x}\right|_{x=0}$ must diverge at $t=0$, forcing $\varphi(x, 0)$ to be discontinuous at $x=0$. With the introduction of our kinetic layer and its associated boundary conditions, we can take $\varphi(x, 0)=\varphi_{0}$, for all $x \geq 0$, resulting in a continuous concentration field.

For long times, we found that convection dominated the motion of the interface and that its velocity was always in the opposite direction of the drift-which is to be expected. However, although switching the sign of the convection switches the sign of the interface's long-time velocity, we discovered that the interface speed is modified. In particular, for fixed kinetic rates and layer capacity, a convective drift away from $X$ resulted in a long time interface velocity that was independent of the initial condition $\varphi_{0}$, whereas a drift into $X$ drove the interface to eventually move with a constant velocity that was $\varphi_{0}$-dependent. Interestingly, the introduction of a drift into the interface makes possible the existence of steady state solutions. When drift is away from the interface, no steady state solutions are found; instead, traveling waves propagate away from the interface under certain conditions (see (4.2)). Given that our current understanding of surface growth under the effects of convection is somewhat incomplete [24], we think that these results may be important in the context of step-bunching instabilities that arise due to a forced adatom drift [16].

In summary, our study shows that the growth of an interface can exhibit a variety of behaviors, depending on the interplay between kinetics, diffusion, and convection. There are many important extensions to our work. For example, we have not analyzed the radial growth of circular/spherical precipitates. In these cases, strain effects at a curved interface can render the dissociation rate $k_{\text {off }}[R(t)]$ a function of the precipitate radius $R(t)$. Including such effects with diffusion and convection may lead to additional nonmonotonic interface dynamics. Finally, an interesting computational application of (2.16) would be to apply the integral equation method in [15] to a step model of surface growth that includes the effects of convection, kinetics, and layer capacity.

\section{Appendix.}

6.1. Limit result of $\boldsymbol{x} \rightarrow \mathbf{0}$ in (2.15). Equation (2.16) is obtained using

$$
\begin{aligned}
I & \equiv \lim _{x \rightarrow 0} \int_{0}^{t} \frac{x+X(t)-X(t-\tau)}{2 \tau} e^{-(x+X(t)-X(t-\tau)-U \tau)^{2} / 4 \tau} \varphi_{s}(t-\tau) \frac{\mathrm{d} \tau}{\sqrt{\tau}} \\
& =\int_{0}^{t} \frac{X(t)-X(t-\tau)}{2 \tau} e^{-(X(t)-X(t-\tau)-U \tau)^{2} / 4 \tau} \varphi_{s}(t-\tau) \frac{\mathrm{d} \tau}{\sqrt{\tau}}+\sqrt{\pi} \varphi_{s}(t) .
\end{aligned}
$$

Copyright $@$ by SIAM. Unauthorized reproduction of this article is prohibited. 
This last equality is shown by splitting the range of integration so that $I=I_{1}+I_{2}$ :

$$
I_{1}=\int_{0}^{x} \frac{x+X(t)-X(t-\tau)}{2 \tau} \exp \left[\frac{-(x+X(t)-X(t-\tau)-U \tau)^{2}}{4 \tau}\right] \varphi_{s}(t-\tau) \frac{\mathrm{d} \tau}{\sqrt{\tau}}
$$

and

$$
I_{2}=\int_{x}^{t} \frac{x+X(t)-X(t-\tau)}{2 \tau} \exp \left[\frac{-(x+X(t)-X(t-\tau)-U \tau)^{2}}{4 \tau}\right] \varphi_{s}(t-\tau) \frac{\mathrm{d} \tau}{\sqrt{\tau}} .
$$

Using the substitution $y=x / \sqrt{\tau}$, we obtain

$$
\begin{aligned}
I_{1}= & \int_{\sqrt{x}}^{\infty}\left(1+\frac{X(t)-X\left(t-x^{2} / y^{2}\right)}{x}\right) \\
& \times e^{-\frac{1}{4}\left[y+\frac{y}{x}\left(X(t)-X\left(t-x^{2} / y^{2}\right)\right)-U x / y\right]^{2}} \varphi_{s}\left(t-\frac{x^{2}}{y^{2}}\right) \mathrm{d} y .
\end{aligned}
$$

When $x \ll 1$,

$$
I_{1} \sim \int_{0}^{\infty}\left(1+\frac{\dot{X}(t) x}{y^{2}}\right) e^{-\frac{1}{4}\left[y+\frac{x}{y}(\dot{X}(t)-U)\right]^{2}} \varphi_{s}\left(t-\frac{x^{2}}{y^{2}}\right) \mathrm{d} y .
$$

Since $0<x / y<\sqrt{x}$, when $x \rightarrow 0, x / y \rightarrow 0$ also. Note that the lower limit of the second term in $(6.5) \sim \int_{0} x / y^{2} \mathrm{~d} y=O(x / y) \rightarrow 0$ and $I_{1}$ is convergent. Hence,

$$
\begin{aligned}
I_{1} & \rightarrow \varphi_{s}(t) \int_{0}^{\infty} e^{-\frac{y^{2}}{4}} \mathrm{~d} y \\
& =\sqrt{\pi} \varphi_{s}(t) .
\end{aligned}
$$

Now consider $I_{2}$. In the exponent, $x / \sqrt{\tau}=O(\sqrt{x})$ near the lower limit. Furthermore, the contribution from $\frac{x}{\tau^{3 / 2}}$ (upon integration) is also $O(\sqrt{x})$. Therefore when $x \rightarrow 0$,

$$
I_{2} \rightarrow \int_{0}^{t} \frac{X(t)-X(t-\tau)}{2 \tau} e^{-(X(t)-X(t-\tau)-U \tau)^{2} / 4 \tau} \varphi_{s}(t-\tau) \frac{\mathrm{d} \tau}{\sqrt{\tau}} .
$$

6.2. Details of numerical method. Equations (2.9)-(2.12) were solved numerically using first and second order finite differences in time and space, respectively:

$$
\begin{array}{ll}
\left.\varphi_{0}^{m+1}=\varphi_{0}^{m}+\frac{\Delta t}{\varepsilon}\left[-\frac{\varphi_{0}^{m}}{\eta}+\left(\frac{-3 \varphi_{0}^{m}+4 \varphi_{1}^{m}-\varphi_{2}^{m}}{2 \Delta x}\right)-\varphi_{0}^{m} U-v_{0} U\right]\right] & \text { if } \varepsilon>0, \\
\varphi_{0}^{m+1}=\frac{4 \eta \varphi_{1}^{m}-\eta \varphi_{2}^{m}-2 \eta v_{0} U \Delta x}{3 \eta+2 \Delta x+2 \eta U \Delta x} & \text { if } \varepsilon=0, \\
Y^{m}=\frac{v_{0}}{\eta} \varphi_{0}^{m} & \\
X^{m+1}=X^{m}+Y^{m} \Delta t &
\end{array}
$$

Copyright (c) by SIAM. Unauthorized reproduction of this article is prohibited. 
where $\varphi_{n}^{m}$ is the numerical solution at position $n \Delta x$ and time $m \Delta t, \Delta x$ is the grid spacing, $\Delta t$ is the time step, and $X^{m}$ and $Y^{m}$ are the interface position and velocity at the $m$ th time step. For $n=1,2, \ldots, N-1$, updates to the bulk solution were made in a Gauss-Seidel-type fashion, using the latest values available:

$$
\varphi_{n}^{m+1}=\varphi_{n}^{m}+\Delta t\left[\left(Y^{m}-U\right)\left(\frac{\varphi_{n+1}^{m}-\varphi_{n-1}^{m+1}}{2 \Delta x}\right)+\left(\frac{\varphi_{n+1}^{m}-2 \varphi_{n}^{m}+\varphi_{n-1}^{m+1}}{\Delta x^{2}}\right)\right] .
$$

The value $\varphi_{N}^{m}$ is not modified by the numerical scheme.

When $\varepsilon=0$ in (2.9)-(2.12), our equations become identical to those in [8] if the sign of the dependent variable is switched and the independent variable is shifted back to the lab frame. Using the derived similarity solutions in [8], we performed a validation study of our code. For completeness, here we reproduce these similarity solutions:

$$
\begin{aligned}
\varphi(x, t) & =f(\xi)+\eta g(\xi) / \sqrt{t}, \quad \xi=x / \sqrt{t}, \\
X(t) & =\beta \sqrt{t}
\end{aligned}
$$

where

$$
\begin{aligned}
& f(\xi)=\frac{\beta}{2} e^{\beta^{2} / 4} \int_{\xi}^{\beta} e^{-y^{2} / 4} \mathrm{~d} y, \\
& g(\xi)=\frac{\beta}{2}\left(\frac{\beta}{2} e^{-\xi^{2} / 4} \int_{\xi}^{\beta} e^{y^{2} / 4} \mathrm{~d} y-e^{\left(\beta^{2}-\xi^{2}\right) / 4}\right) .
\end{aligned}
$$

For the far field condition $\varphi(x, t) \rightarrow-1-v_{0}$ as $x \rightarrow \infty, \beta$ is found from solving

$$
\beta e^{\beta^{2} / 4} \int_{\beta / 2}^{\infty} e^{-y^{2}} \mathrm{~d} y=1+v_{0} .
$$

We started our numerical method from $t=1$, using $\varphi(x, 1)$ and $X(1)$ from (6.11) and (6.12) as initial conditions. The final integration time was $t=2$; comparisons were made with the exact solutions $\varphi(x, 2)$ and $X(2)$, again coming from (6.11) and (6.12). Using $\eta=0.1, v_{0}=-0.2$, and a domain size $>50$, we obtained first and second order convergence in time and space: see Figure 6.1. In our simulations, typical scheme parameters used were $\Delta x \sim 0.01, \Delta t \sim \sigma \Delta x^{2}$, where $\sigma \sim 0.1-0.5$. Note that the initial conditions for which (6.11) and (6.12) are exact are not constant in $x$, and so $X(t)=\beta \sqrt{t}$ is predicted for all $t$, in contrast to (3.6). 

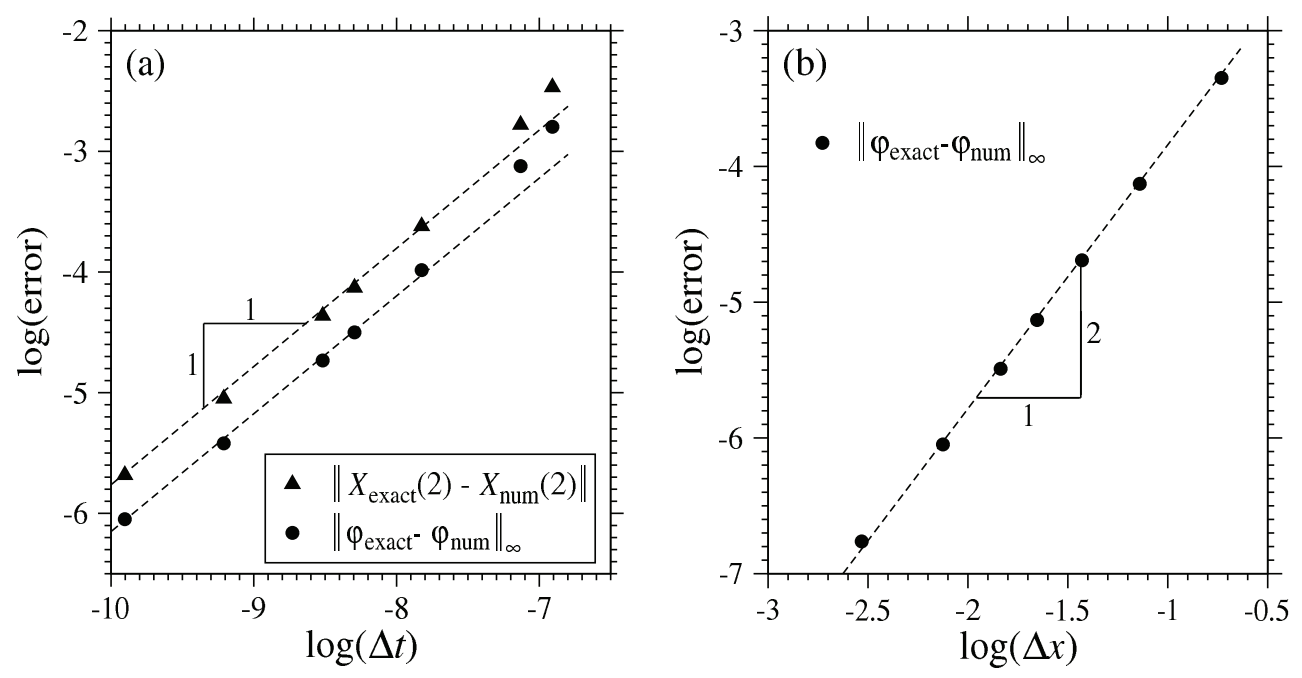

FIG. 6.1. Convergence of the finite difference numerical scheme given by (6.9) and (6.10). Exact solutions were found by solving (6.11)-(6.14), as in [8]. (a) For fixed $\Delta x=0.0477$, errors in $\varphi$ and $X$ scale as $O(\Delta t)$ as $\Delta t \rightarrow 0$. (b) For $\Delta t=10^{-4} \ll \Delta x^{2}$, the error in $\varphi$ scales as $O\left(\Delta x^{2}\right)$.

\section{REFERENCES}

[1] E. Bansch, F. Hauser, O. Lakkis, B. Li, And A. Voigt, Finite element method for epitaxial growth with attachment-detachment kinetics, J. Comput. Phys., 194 (2004), pp. 409-434.

[2] M. Z. BazAnt And T. M. Squires, Induced-charge electro-kinetic phenomena: Theory and microfluidic applications, Phys. Rev. Lett., 92 (2004), 066101.

[3] C. W. Beverung, C. J. Radke, And H. W. Blanch, Protein adsorption at the oil/water interface: Characterization of adsorption kinetics by dynamic interfacial tension measurements, Biophys. Chem., 81 (1999), pp. 59-80.

[4] W. K. Burton, N. Cabrera, and F. C. Frank, The growth of crystals and the equilibrium structure of their surfaces, Philos. Trans. Roy. Soc. London Ser. A., 243 (1951), pp. 299358.

[5] H. S. Carslaw and J. C. Jaeger, Conduction of Heat in Solids, The Clarendon Press, Oxford University Press, New York, 2004.

[6] T. Chou, Stochastic entry of enveloped viruses: Fusion versus endocytosis, Biophys. J., 93 (2007), pp. 1116-1123.

[7] T. Chou And M. R. D'Orsogna, Multistage adsorption of diffusing macromolecules and viruses, J. Chem. Phys., 127 (2007), 105101.

[8] J. N. Dewynne, S. D. Howison, J. R. Ockendon, and W. Xie, Asymptotic behavior of solutions to the Stefan problem with a kinetic condition at the free boundary, J. Austral. Math. Soc. Ser. B, 31 (1989), pp. 81-96.

[9] H. Diamant, G. Ariel, and D. Andelman, Kinetics of surfactant adsorption: The free energy approach, Colloids and Surfaces A, 183-185 (2001), pp. 259-276.

[10] J. D. Evans and J. R. KIng, Asymptotic results for the Stefan problem with kinetic undercooling, Quart. J. Mech. Appl. Math., 53 (2000), pp. 449-473.

[11] H. Gao, W. Shi, and L. B. Freund, Mechanics of receptor-mediated endocytosis, Proc. Natl. Acad. Sci. USA, 102 (2005), pp. 9469-9474.

[12] B. P. Graham, K. Lauchlan, and D. R. Mclean, Dynamics of outgrowth in a continuum model of neurite elongation, J. Comput. Neurosci., 20 (2006), pp. 43-60.

[13] W. Hong, H. N. Lee, M. Yoon, H. M. Christen, D. H. Lowndes, Z. Suo, and Z. Zhang, Persistent step-flow growth of strained films on vicinal substrates, Phys. Rev. Lett., 95 (2005), 095501.

[14] S. Howison And X. WeIQIng, Kinetic undercooling regularization of supercooled Stefan problems, in Mathematical Models for Phase Change Problems, Internat. Ser. Numer. Math. 88, Birkhäuser, Basel, 1989, pp. 227-237.

[15] J. Huang, M.-C. LaI, And Y. XIAng, An integral equation method for epitaxial step-flow 
growth simulations, J. Comput. Phys., 216 (2006), pp. 724-743.

[16] A. Latyshev, A. Aseev, A. Krasilnikov, and S. Stenin, Transformations on clean Si (111) stepped surface during sublimation, Surf. Sci., 213 (1989), pp. 157-169.

[17] S.-Y. Lin, K. McKeigue, And C. Maldarelli, A diffusion-limited interpretation of the induction period in the relaxation in surface tension due to adsorption of straight chain, small polar group surfactants: Theory and experiment, Langmuir, 9 (1991), pp. 1055-1066.

[18] M. Mulqueen, K. J. Stebe, and D. Blankschtein, Dynamic interfacial adsorption in aqueous surfactant mixtures: Theoretical study, Langmuir, 17 (2001), pp. 5196-5207.

[19] M. Sato And M. Uwaha, Growth law of step bunches induced by the Ehrlich-Schwoebel effect in growth, Surf. Sci., 493 (2001), pp. 494-498.

[20] T. P. Schulze, P. Smereka, And W. E, Coupling kinetic Monte-Carlo and continuum models with application to epitaxial growth, J. Comput. Phys., 189 (2003), pp. 197-211.

[21] T. M. Squires And M. Z. BAZAnt, Induced-charge electro-osmosis, J. Fluid Mech., 509 (2004), pp. $217-252$.

[22] S. Stoyanov And V. Tonchev, Properties and dynamic interaction of step density waves at a crystal surface during electromigration affected sublimation, Phys. Rev. B, 58 (1998), pp. 1590-1600.

[23] A. F. H. Ward And L. TordaI, Time-dependence of boundary tensions of solutions I. The role of diffusion in time-effects, J. Chem. Phys., 14 (1946), pp. 453-461.

[24] K. Yagi, H. Minoda, and M. Degawa, Step bunching, step wandering and faceting: Selforganization at Si surfaces, Surf. Sci. Rep., 43 (2001), pp. 45-126.

[25] C. Ybert And J. M. Di Meglio, Study of protein adsorption by dynamic surface tension measurements: Diffusive regime, Langmuir, 14 (1998), pp. 471-475.

Copyright (c) by SIAM. Unauthorized reproduction of this article is prohibited. 